\title{
ARE UBIQUITOUS TECHNOLOGIES THE FUTURE VEHICLE FOR TRANSPORTATION PLANNING : AN ANALYSIS ON VARIOUS METHODS FOR ORIGIN DESTINATION
}

\author{
Tapas Saini, S.V.Srikanth \& Amritanshu Sinha \\ Centre for Development of Advancement Computing (C-DAC) \\ Hyderabad, India
}

\begin{abstract}
Origin Destination has become a crucial aspect in long term transportation planning. For Origindestination estimations, wide variety of methods can be used. Conventional methods like home surveys \& roadside monitoring are slow \& less effective. Bluetooth \& CCTV cameras are also feasible methods for doing OD study, but have their own downsides. At present, this information contributes to very less percentage of data collection. Ubiquitous technologies like mobile phones being deployed in the proposed research is estimated to enhance the data collection and provide a quick \& effective OD estimation. In this paper we discuss how technology becomes the future vehicle for OD.
\end{abstract}

\section{KEYWORDS}

Origin Destination matrix, CCTV, Bluetooth, GPS, Mobile phone

\section{INTRODUCTION}

In this era of modernization the cities of India are quickly getting modernized. Mass number of people keeps moving from rural to urban areas because of good amenities. The migration of rural population to urban areas in search of better job prospects has made cities densely populated. Eight cities have population more than 3 million [1]. With rising incomes in urban areas, car loans proliferate and the auto industry churns out low-cost cars to nudge them off their motorcycles, Indians are rushing headlong to get behind the wheel. Indians bought 1.5 million cars in 2007, more than double of that in 2003. This increase in cars is making our roads narrow, and increases congestion. Here the need for better transportation planning arises. One of the most crucial requirements for the transportation planning is on arriving at the traffic pattern between various zones through Origin-Destination matrix (ODM) estimation [2]. A large number of people commute every day inside the city for different purposes. Of these commuters, majority are employees. These employees move every day to $\&$ fro from different points in the city. Their travel modes comprise from two wheelers to heavy public transport vehicles. Origin \& Destination is the start \& end through these travels.

An origin-destination study is used to determine travel patterns of traffic on an installation during a typical day. They are useful in assisting long-range transportation planning, especially when there are substantial changes anticipated in the installation mission or strength. At the starting of 
day people starts from a location say home (zone). These are called as ORIGINS. Their journey ends to their workplaces (zone), which are called as DESTINATIONS

Origin-destination matrices (OD-matrices) show the number of trips from every zone in the area under investigation to all the other zones. A zone is an area containing a number of inhabitants, a number of work places and other characteristics. Usually, a zone corresponds to a city, a village or a municipality. The matrices are also called demand matrices, based on the meaning of the data [3].

Every day, large number of people move from one place to other in city. This mass movement is mapped to a two dimensional figure as origin-destination matrix. Each cell of the matrix represents the trip counts for a specific combination of origin and destination. Origin-destination estimations are done for decades through conventional methods for instance house hold surveys, road side manual counts etc. Further methods include approaches such as entropy modeling, "best fit" approaches, as well as traffic modeling approaches which are nominally dependent on an "Old" (Outdated) matrix' [4]. Medina and Taft worked on Traffic Matrices estimation techniques like linear programming \& statistical approaches [5]. Ilsoo Yun and Byungkyu (Brian) Park developed a genetic algorithm (GA)-based dynamic O-D matrix estimation method applicable for a large-scale network [6]. These conventional methods are slow \& outdated mechanisms which doesn't provide fast \& economical results. New technological methods can be used for getting fast accurate results. Here we explore different ways of raw data collection for the purpose of estimating origin destination matrices.

\section{ORIGIN DESTINATION ESTIMATION METHODS}

\subsection{House hold surveys}

Household surveys are traditional techniques for data collection. Data collection is done by visiting individual homes for each individual's travel areas. This survey is done usually once in decades. Survey men keep a questionnaire, which is prepared for getting input data. People are queried for how $\&$ when they travel different places in the city. These travel patterns may change on weekends, rainy seasons etc. After recording the details in questionnaire, data is fed into the on line database. Figure 1 shows the estimation of OD matrices from database. The results are generated later as origin-destination matrices.

\subsubsection{Pros \& Cons}

- Easy to do as it just requires a simple questionnaire.

- Problem of underreporting, respondents are dependent on their memories [6].

- The cost of data collection is high. It requires lot of manpower to do.

- It is a slow process, as from collection to feeding into system takes couple of days. This results in more time consumption to show final results. If this survey is conducted to large scale it may take couple of months to get results.

- When we get the results, they may become obsolete. 


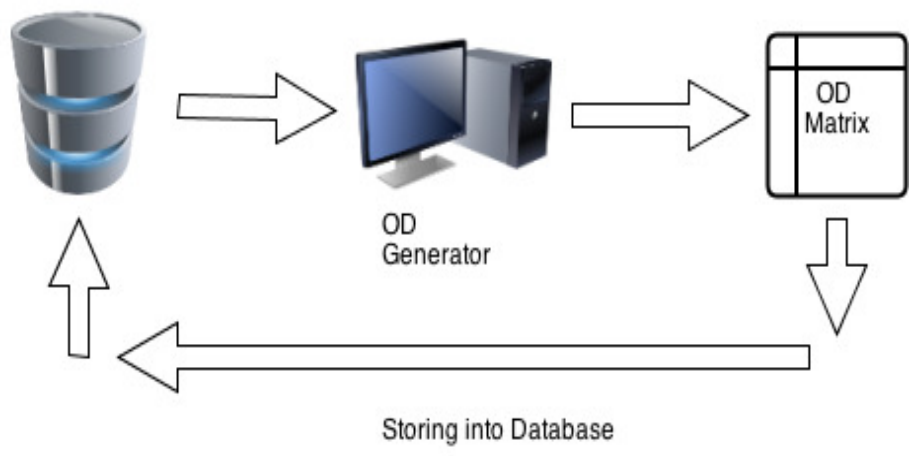

Figure 1: OD calculations

\subsection{Using Cameras}

Using close circuit TV cameras is another methodology to collect raw data. These cameras can be installed at the traffic junctions. Pictures of traffic are taken from time to time, mostly in morning $\&$ evening at peak hours. These pictures can then be sent to the web server located remotely through Internet. Image processing techniques are applied over this pictorial data for results.

\subsubsection{Pros \& Cons}

- This is a good mechanism to measure traffic density.

- Numerous image-processing tools can be used to find out the percentage of traffic density on traffic junctions.

- Traffic counting can also be done as a bye product.

- Cannot be used for OD estimations completely.

- Difficult to re identify same commuters at other junctions.

- The progress of license plate recognition system can help but its still a long way to go.

\subsection{Using GPS probes in public vehicles}

GPS enabled probes is another method for data calculation. These probes are embedded systems with GPS \& GPRS Internet connectivity. The probes log GPS points of the vehicles periodically [9]. After logging locations, the data can be uploaded on the remote web server either in real time or non-real time basis. This method is commonly used in public transport buses mainly used to track the bus routes. Eisenman and List [4] worked on the idea of using probe data for OD matrices. This system is already deployed in large number of cities in India. State level transport corporations are deploying these probes in their buses for monitoring movement of their buses

\subsubsection{Pros \& Cons}

- Public transport vehicles move in a predefined route. Their origin \& destination is predefined. 
- Only public transport data cannot be used for OD study as this data accounts for a small percentage of total number of vehicles on the road. Most of the vehicles are private owned vehicles, which do not necessarily follow same route as of public vehicles.

- These probes cannot be put in private vehicles.

- We cannot judge the movement of complete traffic on the basis of this data.

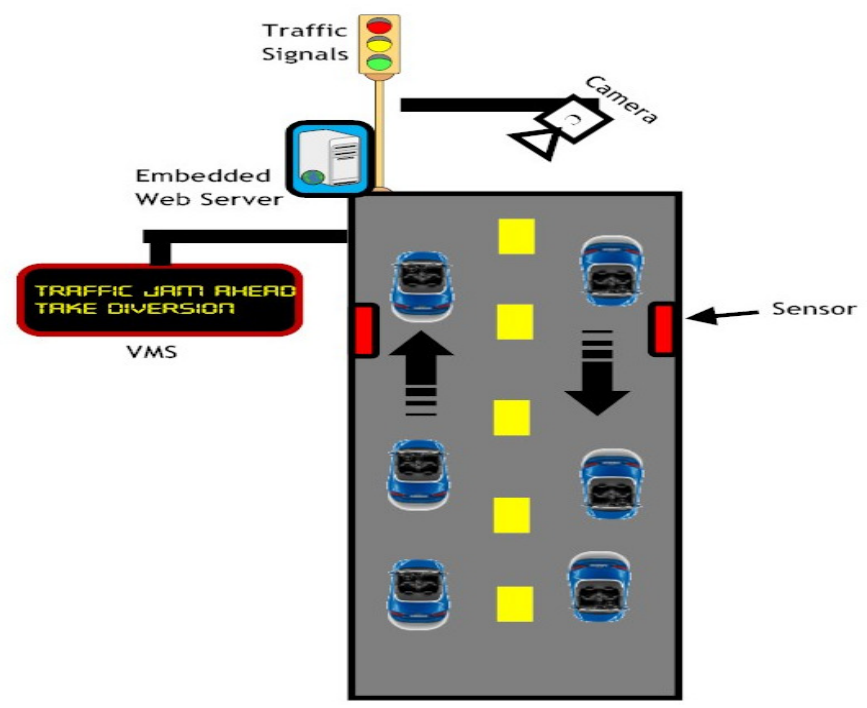

Figure 2: Using Cameras \& Bluetooth

\subsection{Bluetooth}

Bluetooth is a low range \& low power technology for Adhoc wireless communication. Bluetooth is playing an important role in wireless communications in personal area networks. Bluetooth works on unlicensed radio frequency band, which does not require any prior permission from regulators. Bluetooth is incorporated in wide range of devices like mobile phones, cars, bluetooth headsets etc. While moving in traffic people use bluetooth for listening to music through their mobile phones, cars and other variety of purposes.

For OD purposes, we can sniff presence of these bluetooth devices. Every bluetooth device has a mac address, which is unique worldwide. High-powered bluetooth sniffers can be placed on traffic junctions. These bluetooth sniffers can sniff the presence of movable bluetooth systems on the roads. The proposed bluetooth sniffers sniff the mac addresses of the bluetooth devices hence getting the unique id for origin-destination estimations. MAC address obtained should be encrypted before analysis for OD study.

\subsubsection{Pros \& Cons}

- The encrypted MAC address doesn't pose any privacy threat.

- There is decent increase in use of Bluetooth enable devices. 
- Bluetooth is still not installed in majority of basic phones.

- The bluetooth of mobile device has to be switched on at the time of sniffing.

- People may keep their bluetooth devices in hidden mode. As a result, device will be invisible to sniffer.

\subsection{Using Mobile phones}

Mobile phone has become a basic necessity in the country. They are ubiquitous in today's world. This power of mobile can be harnessed to get raw data for OD study. Millions of people carry mobiles while moving inside city. If we get their travel points in the city through mobile phone, then we can easily estimate their movements with condition that their privacy has to be maintained [10][11]. Data about the movement of mobiles can be taken through two ways:

\subsubsection{Active data collection}

Mobiles themselves do this active data collection. We need smart mobile phone for doing this collection. Applications can be developed for mobiles, which harness the power. Varieties of mobile OSs are available on which custom applications can be programmed. Examples are Android, IOS, symbian etc... [14]. These smart mobiles need to have either GPS or GPRS facility. GPS can be used to record latitude \& longitude values of the mobile periodically. The periodicity can be set by the user depending on his choice. Periodicity can also be varied depending on the battery level of mobile phone.

This data can be written into a file into mobile storage by developed application. In the absence of GPS connectivity mobile service providers can be queried for latitude \& longitude values. The mobile service provider calculates the approximate position of the mobile device. This data is requested through GPRS channel. Again this data is logged into a file in the phone storage. Later this file has to be uploaded on the web server for further analysis. Uploading file on web server requires GPRS Internet connection. Figure 1 shows the above behavior. This data can be analyzed later by the OD generator to generate OD matrices.

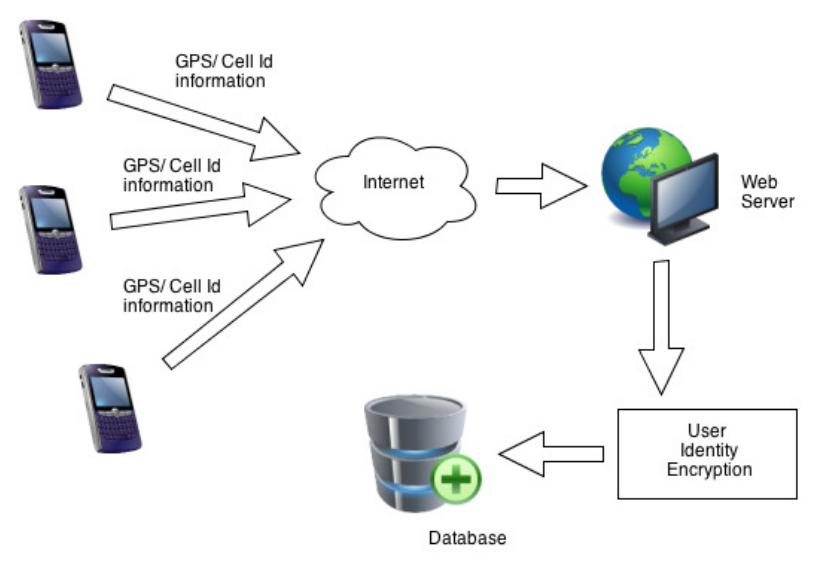

Figure 3: Active Data collection from mobile phones 
International Journal of Ad hoc, Sensor \& Ubiquitous Computing (IJASUC) Vol.4, No.6, December 2013

\subsubsection{Pros \& Cons}

- Doesn't need help of mobile service providers.

- Get accurate location of mobile through GPS device on mobile phone, if available.

- Extra data like speed, altitude of mobile phone can be recorded.

- With GPS, it is impossible to perform positioning indoors or in other areas shielded from GPS signals,

- GPS device drains mobile battery faster, needs battery power saving mechanisms.

- Requires active participation from people.

- Covers less percentage of people, as it needs a smart phone for application to sit onto it.

\subsubsection{Passive data collection}

This data collection is done by mobile service providers. Mobile service providers have preinstalled infrastructure. They use their high towers for providing signal services to the user's mobile phones [12][13]. The mobile service providers keep track of the users call records as Call Detailed Records (CDR). Whenever user makes a call from his mobile, mobile service provider estimates his location and stores into its call data records. These call data records can be extremely useful in estimating users' travel paths. The mobile service providers can be hesitant in providing CDRs. They may take a back step as this may breach the privacy of mobile phone users [8]. As a solution, the mobile numbers can be taken in encrypted format. After encryption, the user's identity is hidden and this data can be converted to OD matrices [15].

\subsubsection{Pros \& Cons}

- It doesn't require any special infrastructure to setup.

- It can be done not only for smart phones but any basic mobile also.

- With mobile phones in wide use, this methodology covers a big percentage of users.

- As data is encrypted, privacy is maintained.

- Mobile service providers hesitate to provide raw data of users due to privacy reasons.

- GPS data from mobile operators is less accurate than GPS device in mobile phones. 


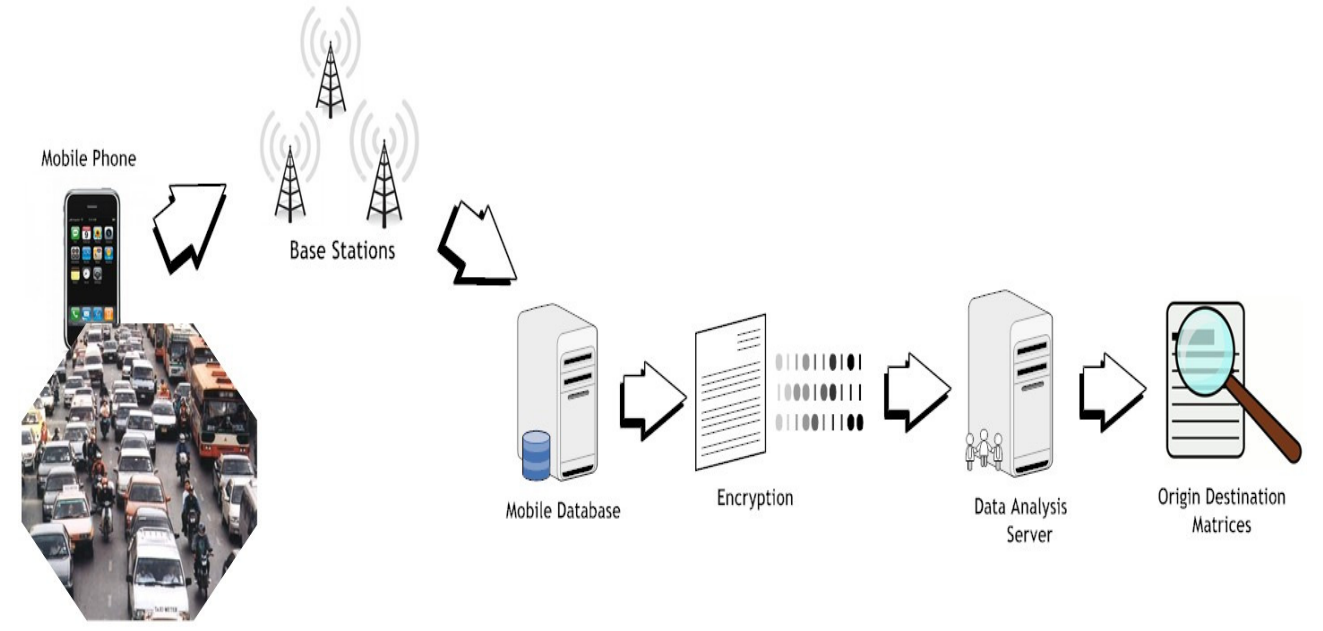

Figure 4: Passive Data Collection

There is yet another way to collect the mobile users data. Instead of call data records, mobile service provider can provide Application Programming Interfaces (APIs) to query their database. These databases may have query limit. Day to day random \& limited number of users can be extracted from the database. The raw data from these queries should contain the latitude \& longitude of the mobile users. Again this data has to be processed with user identities encrypted.

Figure 5 shows a blend of various methodologies for OD collection. Thus we can see that OD can be estimated in different ways but growing use of technology looks promising in getting fast \& accurate results for a better urban transportation planning.

\section{SUMMARY}

To summarize, initially we discussed about the traditional ways of collecting data for estimating origin-destination matrices. Traditional methods like household surveys are time consuming \& ineffective. Cameras at junctions are good for finding traffic density but don't help in re discovering same vehicles at other junctions, which is critical requirement for OD study. Using GPS probes in buses is another method for collecting data, but it cannot be used for private vehicles, which contributes to the maximum amount of vehicles on the road. Bluetooth is also a feasible method, where we can sniff personal bluetooth devices through high range bluetooth sniffers. But low penetration of bluetooth among basic mobile users, restricts OD estimations.

Finally, Mobile phones can be used for finding unique users anywhere in the city. These mobiles movement tracking is not restricted to junctions. We discussed two ways for collecting raw data through mobiles. Active method in which mobile itself collects data through its GPS sensors. In the absence of GPS, data can be requested from mobile service provider. This data can be logged in a file on mobile \& later forwarded to the web server. Passive method consists of taking raw data from mobile service providers. This raw data is present with mobile service providers in the form of call data records. In absence of CDRs, the MSPs database can be queried for random users data. Note that user's identity has to be encrypted before analysis. Hence combinations of all these methods can also be used to find \& validate mobile user data for final OD estimations. 
International Journal of Ad hoc, Sensor \& Ubiquitous Computing (IJASUC) Vol.4, No.6, December 2013

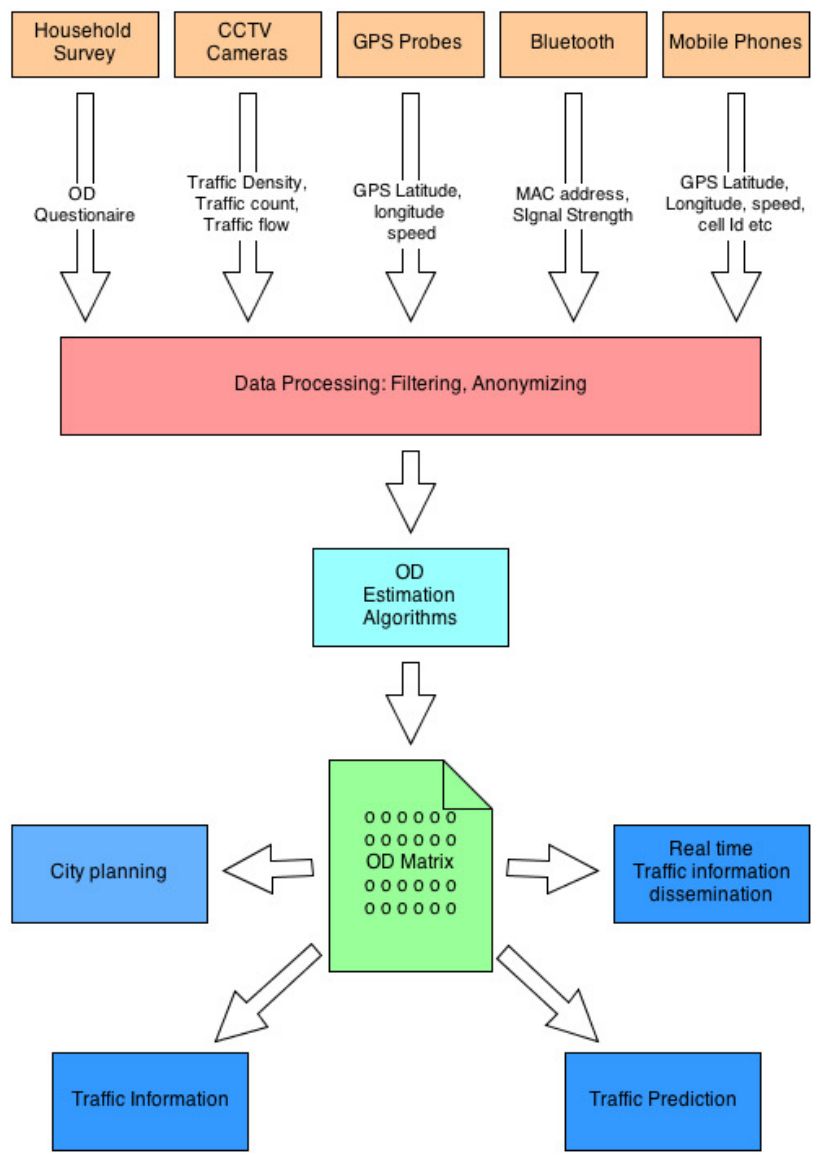

Figure 5: OD estimation through various methodologies

\section{CONCLUSION}

The basic goal of this paper is to discuss about different ways for collecting raw data intended for origin destination estimations. Conventional methods of travel data collection are slow \& ineffective. These methods take lot more manpower to do the surveys. Use of cameras, GPS probes \& bluetooth technologies is feasible, but they contribute to OD data collection in small extent. We propose the use of ubiquitous mobile phones as nearly every family in cities is carrying mobile phones. Ocean of data from these mobile phones can be analyzed to get fast, accurate and up to date OD matrices. At present, this information contributes to $1 \%$ of the data. Ubiquitous technologies being deployed in the proposed research is estimated to enhance the same to $30 \%$. This shows technology is the future vehicle for an effective and efficient urban transportation planning.

\section{ACKNOWLEDGEMENTS}

We are grateful to the Centre for Development of Advanced Computing (C-DAC) for encouraging our efforts in pursuing Research and Development in the areas of Ubiquitous Computing. This work is funded by Department of Electronics \& Information Technology (DeitY), Ministry of Communication \& IT, Govt. of India under the project "Advanced Research in Ubiquitous Computing". 
International Journal of Ad hoc, Sensor \& Ubiquitous Computing (IJASUC) Vol.4, No.6, December 2013

\section{REFERENCES}

[1] Azeem Uddin, "Traffic congestion in Indian cities: Challenges of a rising power" Kyoto of the Cities, Naples, Mar 26-28, 2009

[2] Sharminda Bera 1, K. V. Krishna Rao, "Estimation of origin-destination matrix from traffic counts: the state of the art” European Transport \Trasporti Europei n. 49 (2011): 3-23

[3] Marcel Rieser, “Generating Day Plans From Origin-Destination Matrices”August 2004 ETH Zurich, Switzerland

[4] Stacy M. Eisenman and George F. List, "Using Probe Data to Estimate OD Matrices". 2004 IEEE Intelligent Transportation Systems Conference Washington. D.C., USA, October 36,2004

[5] A. Medina, N. Taft, K. Salamatian, S. Bhattacharyya, C. Diot, "Traffic Matrix Estimation: Existing Techniques and New Directions”. Sprint Advanced Technology Labs. Burlingame, CA.Department of Computer Science, Boston University. Boston MA.University of Paris VI. Paris, France.

[6] Ilsoo Yun and Byungkyu (Brian) Park, "Estimation of Dynamic Origin Destination Matrix: A Genetic Algorithm Approach". Proceedings of the 8th International IEEE Conference on Intelligent Transportation Systems Vienna, Austria, September 13-16, 2005

[7] Shinji Itsubo, "A study of the effectiveness of a household travel survey using GPS-equipped cell phones and a WEB diary through a comparative study with a paper based travel survey" Researcher, Traffic Engineering Division, Ministry of Land, Asahi Tsukuba-City Ibraki-Pref, 305-0031 Japan.

[8] Gary Larkin, “New Privacy Threats”. PC World. Pg 20-22. June 2006

[9] Geoff Rose, "Mobile Phones as Traffic Probes: Practices, Prospects and Issues". Transport Reviews. May 2006

[10] Scott Fraser, "The use of floating cellular telephone data For real-time transportation incident Management”, Thesis, McMaster University,

[11] N. Caceres, J.P. Wideberg, and F.G. Benitez, "Deriving origin-destination data from mobile phone network, " 2007

[12] J. White and I. Wells, “Extracting origin destination information from mobile phone data," in 11th Int. Conf. on Road Transport Information and Control, LONDON, 2002, pp. 30-34

[13] M. H“opfner, K. Lemmer, and I. Ehrenpfordt, "Cellular data for traffic management," in 6th European Congress and Exibition on Intelligent Transport System and Services, 2007

[14] Erik Mellegard, "Obtaining Origin/Destination-matrices from cellular network data”, Master Thesis, Chalmers University of Technology, Sweden, 2011

[15] http://www.airsage.com/

\section{AUTHORS}

Mr. Tapas is a member of the National Ubiquitous Computing Research Resource Center of C-DAC, Hyderabad. He is working in C-DAC Hyderabad for last 7 years. Presently, He is working as Senior Technical Officer. Being a part of embedded systems design \& development team, he holds a significant expertise in the area of Wireless Sensor Networks. Under the umbrella of ubiquitous computing, he is actively involved in the Research in Origin Destination for Urban Transportation Enhancements (ROUTE) project. His area of interest is mobile computing.

Mr. S.V.Srikanth is a member of the National Ubiquitous Computing Research Resource Centre of CDAC, Hyderabad. He has been associated with C-DAC Hyderabad for the last 9 years in the field of Embedded System Design and Ubiquitous Computing. Currently he is designated as Senior Technical Officer. He obtained Masters in Networking and Telecom systems from UK and presently pursuing PhD in the area of Wireless Heterogeneous Networks from JNT University, Hyderabad, India. His areas of interest include Wireless Technologies, Embedded System Design and Mobile Communications.

Mr. Amritanshu Sinha has been working as Intern Engineer with C-DAC Hyderabad for the last one year in the field of Ubiquitous Computing. He has completed his Bachelors in Computer Science from Magadh University, Bihar, India. His area of interest is mobile computing \& embedded systems. 